\title{
Percheron-like artery infarction after transsphenoidal surgery: illustrative case
}

\author{
Lennart W. Sannwald, ${ }^{1}$ Andrea von Helden, Dr med, ${ }^{2}$ Hans-Joachim Wagner, $\operatorname{Dr}$ med, ${ }^{3}$ Dag Moskopp, Dr med, ${ }^{1}$ and \\ Mats L. Moskopp, $\mathrm{PhD}^{1,4}$

\begin{abstract}
${ }^{1}$ Department of Neurosurgery and ${ }^{3}$ Institute for Radiology and Interventional Therapy, Vivantes Friedrichshain Hospital, Charité Academic Teaching Hospital, Berlin, Germany; ${ }^{2}$ Center for Severe Cerebral Injuries, Vivantes Spandau Hospital, Charité Academic Teaching Hospital, Berlin, Germany; and ${ }^{4}$ Institute of Physiology, Medical Faculty Carl
\end{abstract} \\ Gustav Carus, Technische Universität Dresden, Dresden, Germany
}

BACKGROUND The transsphenoidal approach to the skull base has enjoyed increasing popularity for surgery of the sellar region avoiding brain retraction and causing few severe complications. While vitally important vessels in this region show a high degree of variability, some anatomical variants might be involved in characteristic complications.

OBSERVATIONS We present the case of a 40-year-old female patient with acromegaly due to a pituitary adenoma that was transsphenoidally operated. Postoperatively, the patient presented with bilateral unresponsive mydriasis, loss of consciousness and tetraparesis. An MRI showed wellcircumscribed bilateral paramedian thalamic infarctions which indicated a rare Percheron-like artery. At 2-year follow-up examination the patient was dramatically improved but with a profound impact on her ability to interact with the world.

LESSONS The basilar artery or perforators might be injured during dissection of suprasellar lesions. This vascular territory is essential to interaction of the brain with the outside world. We conclude that we will approach future suprasellar adenomas strictly intracapsularly.

https://thejns.org/doi/abs/10.3171/CASE21612

KEYWORDS Percheron; pituitary surgery; transsphenoidal surgery; bithalamic infarctions

Transsphenoidal surgery is an established procedure avoiding both craniotomy and direct manipulation of brain tissue by use of a transnasal approach to skull base lesions such as pituitary adenomas, skull base meningiomas, metastases, and craniopharyngiomas. Over the last decades, the transsphenoidal approach has enjoyed increasing popularity for surgery of the sellar region as it helps avoiding brain retraction and causes few severe complications such as cerebrospinal fluid fistula, postoperative meningitis, and injury to the internal carotid artery. However, this approach usually enables only limited or indirect access to suprasellar extensions of sellar lesions, while a suprasellar extension may increase contact to delicate structures at the base of the brain such as the optic chiasm, circle of Willis, cranial nerves III, IV, VI, and the basilar artery.
Here, we report a rare but serious complication of transsphenoidal surgery of a pituitary macroadenoma in a 40-year-old patient with acromegaly. In addition, this case illustrates the importance of small and highly variable blood vessels for human health and physiology as well as the potential of neurorehabilitation.

\section{Illustrative Case}

\section{Initial Presentation}

A 40-year-old female patient presented with typical symptoms of acromegaly and profuse sweating as well as bitemporal hemianopsia and impaired color vision. Her prior endocrinological history included secondary amenorrhea. Acromegaly was confirmed by laboratory

\footnotetext{
ABBREVIATIONS ADC = apparent diffusion coefficient (MRI sequence); $\mathrm{AOP}=$ artery of Percheron; $\mathrm{cCT}=$ cranial computed tompgraphy; $\mathrm{cMRI}=\mathrm{cranial}$ magnetic resonance imaging; $\mathrm{CPP}=$ cerebral perfusion pressure; $\mathrm{CSF}=$ cerebrospinal fluid; $\mathrm{CT}-\mathrm{A}=$ computed tomography angiography; $\mathrm{DWI}=$ diffusion weighted image (MRI sequence); FLAIR = fluid-attenuated inversion recovery (MRI sequence); $h \mathrm{GH}=$ human growth hormone; ICP = intracranial pressure; IGF = insulinlike growth factor; $\mathrm{M}: \mathrm{F}=$ male vs female; oGTT = oral glucose tolerance test; $\mathrm{P} 1=$ pre-communicating segment of the posterior cerebral artery; $\mathrm{PCA}=$ posterior cerebral artery; PCOM = posterior communicating artery.
}

INCLUDE WHEN CITING Published February 7, 2022; DOI: 10.3171/CASE21612.

SUBMITTED October 29, 2021. ACCEPTED November 29, 2021.

(c) 2022 The authors, CC BY-NC-ND 4.0 (http://creativecommons.org/licenses/by-nc-nd/4.0/). 

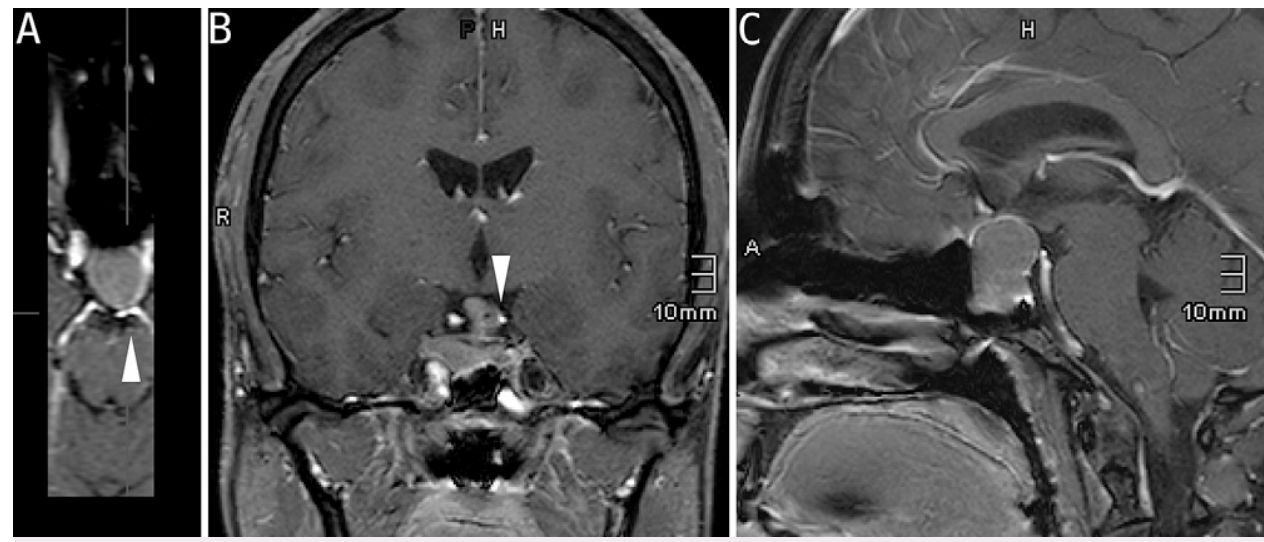

FIG. 1. Initial T1-weighted contrast-enhanced cMRI of pituitary lesion. Please note the proximity between adenoma and vessels as highlighted by arrows.

results (lowest human growth hormone [hGH] after glucose suppression test [oGTT] $31 \mathrm{ng} / \mathrm{ml}$ [ref $<0.4 \mathrm{ng} / \mathrm{ml}]$, insulin-like growth factor (IGF)-1 $893 \mathrm{ng} / \mathrm{ml}$ [ref 72-237 ng/ml]). cMRI showed a pituitary lesion measuring $3 \mathrm{~cm}$ in diameter with contact to the optic chiasm superiorly and the basilar tip posteriorly (Fig. 1). In this situation the indication of a microscopic transsphenoidal resection was given.

\section{Procedure}

Intraoperatively, the lesion showed an atypical rigid consistency. However, step-by-step curettage was performed (by D.M.). The pituitary lesion had no apparent adhesions to the optic chiasm or other neurovascular structures. After an initial debulking an extracapsular resection seemed feasible. During extracapsular preparation of the adenoma an arterial bleeding occurred at the upper left adenoma pole, therefore, only the upper left adenoma pole was coagulated using bipolar and coated thrombin repeatedly. During this period, a short bradycardia was successfully treated using atropine. A small cerebrospinal fluid (CSF) fistula was sealed using abdominal fat and hemostatic agents.

\section{Postoperative Course}

After completion of surgical measures while still inside the operating room, the patient was found with bilateral unresponsive mydriasis. Differential diagnosis at this point included atropine-induced mydriasis. An emergency CCT with CT-A showed no pathological findings. An external ventricular drain with intracranial pressure (ICP) sensor was implanted (initial ICP $4 \mathrm{~mm} \mathrm{Hg}$ ) for ICP and CPP monitoring regarding loss of consciousness and reduction of CSF pressure concerning the CSF fistula. The patient was transferred to the intensive care unit and remained endotracheally intubated. A subsequent MRI showed signs of bilateral, small, well-circumscribed, symmetrical thalamic infarctions (Fig. 2). Despite the small size of the lesions, clinical presentation at this point included loss of consciousness, bilateral unresponsive mydriasis, and tetraparesis. Differential diagnosis included thermal lesion and possible traction of a Percheron-like artery. During the course of the intensive care unit treatment the patient suffered from one episode of meningitis that was treated with antibiotics. Rhinoliquorrhoea due to the previous described CSF fistula was the apparent cause and operatively sealed using fascia of the tensor fascia latae muscle. After 1 month, the patient was transferred to our specialized neurosurgical rehabilitation facility (led by A.v.H.) while the clinical presentation remained unchanged.

During the following months a communicating hydrocephalus occurred and was treated by ventriculoperitoneal shunting.

\section{Two-Year Follow-Up}

At 2-year follow-up the patient had a spastic gait accentuated on the right side. She was vigilant and orientated. General language
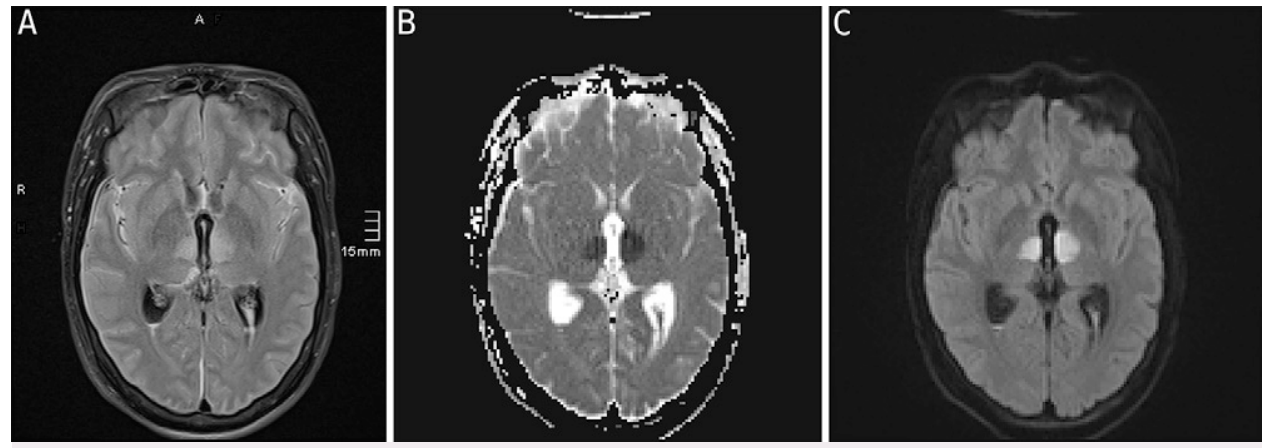

FIG. 2. Representative slices of cMRI 12 hours after operation. Please note signs of bilateral thalamic infarctions (A, FLAIR; B, ADC; C, DWI). Mesencephalic infarction not shown. 


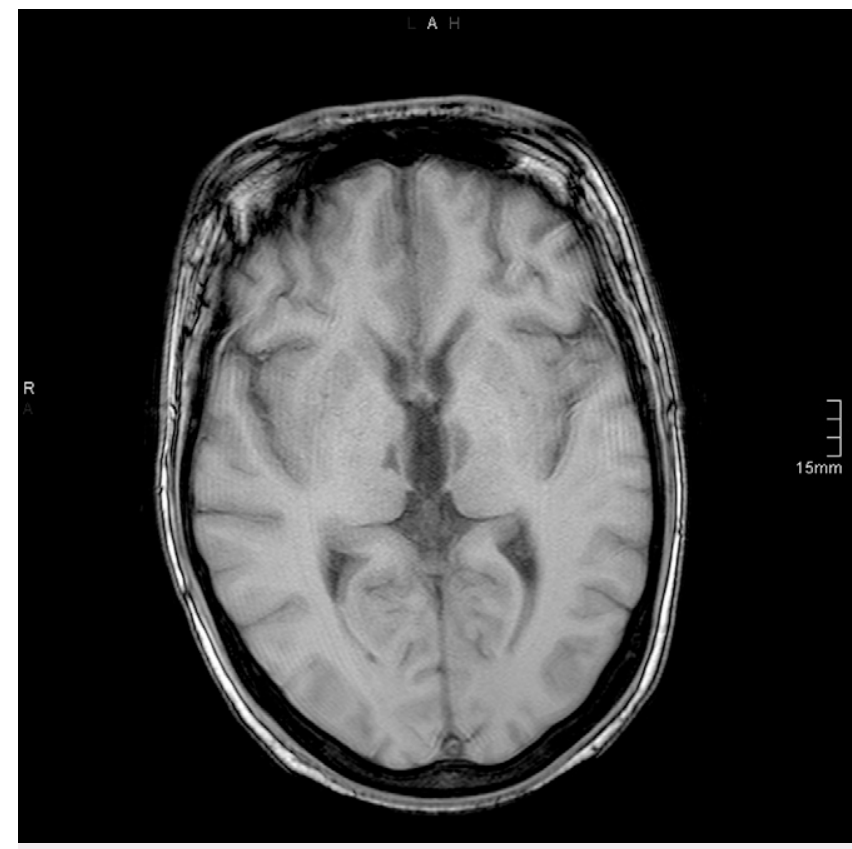

FIG. 3. Representative slice of T1-weighted cMRI 2 years after operation.

understanding and production were preserved. Complex speech impairment included verbal responses that were corrected by the patient herself in a "No ... Yes! Yes!" pattern. Close neuropsychological examination of the patient showed that she had an adequate understanding of complex semantics, was able to repeat tonguetwisting phrases, to rhyme, and displayed contextually adequate humor despite severe constraint in expressing herself. The patient showed short-term memory impairment. She was able to carry out simple mental arithmetic. Deficits of the cranial nerves included vertical gaze palsy, pronounced exotropia of the right eye and reduced vision (left eye, 0.4; right eye, 0.2). The patient denied diplopia and was able to read with both eyes. Coordination of the oral phase of swallowing solid food was impaired while drinking worked without fault. The patient had a high-pitched oscillating phonation. Hearing, smelling, and taste were not impaired. With regard to sensory functions, the patient showed mild pallhypesthesia of the lower limbs. Regarding motor functions, the patient showed a complex movement disorder: a constant motor restlessness without a feeling of inner restlessness, arrhythmic dystonic movements of upper and lower limbs with low amplitude, hyperreflexia, and dysmetria of all extremities. The left half of her body performed motions in an abrupt and accelerated way, whereas her right half remained distinctly slower and assumed a spastic configuration at rest that completely dissolved while in motion. Nevertheless, when given a handshake or pen her movements were fluid and adequate. She was able to independently climb stairs and walk approximately $1,000 \mathrm{~m}$ without a break. Overall, with the ability to walk, climb stairs, dress and undress herself, drink without assistance, and eat with assistance she scored 3 on the Modified Rankin Scale.

Additionally, the patient showed mild remnants of acromegalia and had levels of hGH of $1.6 \mathrm{ng} / \mathrm{ml}$ in oGTT as well as IGF-1 $280 \mathrm{ng} / \mathrm{ml}$. Even though the patient is not normalized according to current guidelines, ${ }^{1}$ these values represent a clear improvement compared with initial presentation. However, she substitutes hydrocortisone, thyroxin as well as desmopressin. She remained amenorrhoic.

A representative MRI of thalamus affection 2 years postoperative is shown in Fig. 3.

\section{Discussion}

\section{Observations}

The blood supply of the thalamus is generally attributed to multiple vessels of $\mathrm{P} 1$ and $\mathrm{P} 2$ segments of the posterior cerebral artery (PCA) as well as the posterior communicating artery (PCOM). As the presence and size of PCOM varies, it comes as no surprise that these small arteries show various, partially individual anatomical variants. The artery of Percheron (AOP) is a rare variant in which a single dominant thalamoperforating artery originating from the PCA P1 segment supplies thalamic paramedian arteries of both sides and may or may not supply the rostral midbrain., ${ }^{2,3}$ However, while Percheron's study was based on anatomical dissections, the AOP can seldomly be directly visualized, neither radiographically nor during surgical preparation. It is most often diagnosed due to indirect signs such as its specific infarction pattern.

In the present case we observed bilateral paramedian thalamus infarctions (Fig. 2) as well as a hyperintense signal in the median mesencephalon in diffusion weighted images. This characteristic infarction pattern in combination with the insistent bleeding and coagulation at the upper left adenoma pole intraoperatively strongly suggests damage to an unpaired left thalamoperforating truncus supplying both thalami due to the proximity of the left posterior adenoma pole to the basilar tip and left P1 (Fig. 1A). This is further supported by the simultaneous occurrence of temporary bradycardia as this can be caused by thermal irritation of parasympathetic fibers of the oculomotor nerve which lies in close proximity to both the PCA and PCOM near the basilar tip. Taken together, this is highly indicative of a lesion of a Percheron-like artery, although it must be emphasized that this is an educated inference based on the phenomenological presentation of the patient.

While isolated AOP infarction is seen in less than $1 \%$ of all strokes, ${ }^{3,4}$ the typical findings in patients with bilateral paramedian thalamic infarctions are described by the triad of altered mental status, vertical gaze palsy, and memory impairment. ${ }^{3}$

The present case is exceptional with regard to the following points. The patient showed a complex motor disorder that is beyond the typical findings of isolated AOP thalamic infarctions without midbrain affection, for example, her right eye nuclear oculomotors nervous palsy and otherwise complex movement disorder. In accordance with oculomotor palsy of the patient a close review of the MRI evidence showed impaired mesencephalic diffusion patterns. However, this does not comprehensively explain the complexity of the observed clinical pattern.

In neuroanatomical classes, the thalamus is often described as a gatekeeper between the outside world and the cortex. Neuropsychological examination showed a complex picture in which intellectual and motor functions associated with the cortex seemed to be partially hidden behind impaired thalamus function, which might lead to a wrong impression of the mental capacity of the patient. Human phenomenal appearance and its perception by others largely depend on language and motor function as intermediary. The issue of a perceived dehumanization of human beings whose ability to adequately interact with the world via language and motor movement was explicitly pointed out by Wittgenstein ${ }^{5}$ in 1914 . This remains a fundamental challenge as 
modern medical intensive care regularly confronts medical personnel with the complexity of human interaction based on the fragility of a patient's motor and language capabilities.

The patient was transferred to a center for neurorehabilitation with loss of consciousness, bilateral unresponsive mydriasis, and tetraparesis. The fact that at the two-year follow-up the patient returned walking and smiling underlines the high potential of neurorehabilitation.

Despite isolated AOP infarctions being among the rarest forms of stroke, to the best of our knowledge only six cases (including the present one) of AOP infarctions after microscopic or endoscopic transsphenoidal resection have been published. ${ }^{6-10}$ Of these six cases, five patients suffered a pituitary adenoma while the sixth case was a 10-year-old girl with a transsphenoidal resection of a craniopharyngioma. Operative approaches were three times endoscopic, two times microscopic, and one time not reported. In four of the six cases CSF leaks occurred during operation. In four cases, but not in the present one, subarachnoid hemorrhage of the basal or interpeduncular cisterns were described after the operation. Interestingly, the sex ratio is $1: 5(\mathrm{M}: \mathrm{F})$. Patient age is reported to be 10 , 36, 40 (twice), 50, and 52 years. Follow-up varied between 6 weeks and 2 years. Outcome was heterogenous from bilateral ptosis (in 2year follow-up of the child) to fatal (36-year-old female, microadenoma as part of multiple endocrine neoplasia 1). A review of more than 1,000 pituitary adenoma cases operated by the senior neurosurgeon (D.M.) showed no similar case. Limitations of this study include that the patient suffered from meningitis and communicating hydrocephalus during her rehabilitations period, which could affect the presentation of her clinical findings.

\section{Lessons}

While injury to the internal carotid artery constitutes the most severe complication of transsphenoidal sellar surgery, posterior suprasellar extension is a risk factor for injury to the basilar tip as well as posterior perforators and should always be kept in mind by transsphenoidal surgeons. This vascular territory is essential to interaction of the brain with the outside world. An extracapsular resection of suprasellar adenomas through a transsphenoidal route poses a significant threat to neurovascular structures at the base of the brain, which can only be visualized partially. Therefore, we concluded that we will approach future suprasellar adenomas strictly intracapsularly. Limiting the resection to the intracapsular adenoma mass leaves a protective layer between surgeon and these neurovascular structures. It is an anatomical paradox that small vessels, which are essential for thalamus blood supply and therefore vitally important arteries, are often not even designated a name due to high interindividual variability. However, the observed infarction pattern is characteristic of an AOP, we did not directly visualize such an artery. Here, we use the term Percheron-like artery. Classification by use of the eponym Percheron provides an accessible term for vitally important but highly variable and unnamed perforating vessels. Awareness of this anatomical paradox, especially in neurosurgery and neurology, is a prerequisite for an understanding of perforating arteries. This is emphasized by the fact that the weighing of risks and benefits that initially led to the operative aim of endocrinological cure in a patient with acromegaly was heavily shifted ex post by a vessel that cannot even be adequately named. Therefore, this case further illustrates the fragility of essential neurological functions, which are, independently of survival, particularly vulnerable in neurosurgical operations. Nevertheless, with time and professional neurorehabilitation, neuroplasticity may enable a significant recovery even in patients with devastating complications.

\section{References}

1. Giustina A, Chanson P, Bronstein MD, et al. A consensus on criteria for cure of acromegaly. J Clin Endocrinol Metab. 2010;95(7): 3141-3148.

2. Percheron G. The anatomy of the arterial supply of the human thalamus and its use for the interpretation of the thalamic vascular pathology. Z Neurol. 1973;205(1):1-13.

3. Lazzaro NA, Wright B, Castillo M, et al. Artery of percheron infarction: imaging patterns and clinical spectrum. AJNR Am J Neuroradiol. 2010;31(7):1283-1289.

4. Ranasinghe KMIU, Herath HMMTB, Dissanayake D, Seneviratne M. Artery of Percheron infarction presenting as nuclear third nerve palsy and transient loss of consciousness: a case report. BMC Neurol. 2020;20(1):320.

5. Wittgenstein L. Vermischte Bemerkungen. In: Schulte J, ed. Wittgenstein - Über Gewißheit. Werkausgabe Band 8. Bemerkungen über die Farben. Über Gewißheit. Zettel. Vermischte Bemerkungen, Suhrkamp Taschenbuch Wissenschaft 508. Frankfurt am Main: Suhrkamp; 1984:451.

6. Sankar T, Souster J, Steinke DE. Bilateral thalamic infarction following transsphenoidal surgery. Can J Neurol Sci. 2008;35(4):522-525.

7. Aryan S, Thakar S, Hegde AS. Artery of Percheron infarction after endoscopic pituitary surgery. Acta Neurochir (Wien). 2016;158(10): 1973-1975.

8. Chau LQ, Levy ML, Crawford JR. Levator palpebrae superioris nuclear palsy in a child with artery of Percheron infarction. BMJ Case Rep. 2018;11(1):10-12.

9. Pereira MR, Hernández FM, Cortés CA. Artery of percheron and endoscopic endonasal surgery: case report and review of the literature. Asian J Neurosurg. 2020;15(3):777-780.

10. Sharma N, Bhattacharjee S, Anne B. A rare complication of pituitary adenoma surgery in a patient with multiple endocrine neoplasia 1 syndrome with two novel genetic mutations. Asian J Neurosurg. 2020;15(4):1020-1023.

\section{Disclosures}

The authors report no conflict of interest concerning the materials or methods used in this study or the findings specified in this paper.

\section{Author Contributions}

Conception and design: M Moskopp, Sannwald, D Moskopp. Acquisition of data: Sannwald, von Helden, D Moskopp, ML Moskopp. Analysis and interpretation of data: Sannwald, D Moskopp, ML Moskopp. Drafting the article: M Moskopp, Sannwald. Critically revising the article: all authors. Reviewed submitted version of manuscript: all authors. Approved the final version of the manuscript on behalf of all authors: M Moskopp. Administrative/technical/material support: $M$ Moskopp, Wagner. Study supervision: ML Moskopp, D Moskopp.

\section{Correspondence}

Mats L. Moskopp: Vivantes Friedrichshain Hospital, Charité Academic Teaching Hospital, Landsberger Allee, Berlin, Germany. s5033630@ msx.tu-dresden.de. 